\title{
On the total mass of closed universes
}

\author{
László B Szabados \\ Wigner Research Centre for Physics, \\ H-1525 Budapest 114, P. O. Box 49, Hungary, \\ E-mail: lbszab@rmki.kfki.hu
}

July 10, 2018

\begin{abstract}
The total mass, the Witten type gauge conditions and the spectral properties of the Sen-Witten and the 3-surface twistor operators in closed universes are investigated. It has been proven that a recently suggested expression $M$ for the total mass density of closed universes is vanishing if and only if the spacetime is flat with toroidal spatial topology; it coincides with the first eigenvalue of the Sen-Witten operator; and it is vanishing if and only if Witten's gauge condition admits a nontrivial solution.

Here we generalize slightly the result above on the zero-mass configurations: $\mathrm{M}=0$ if and only if the spacetime is holonomically trivial with toroidal spatial topology. Also, we show that the multiplicity of the eigenvalues of the (square of the) Sen-Witten operator is even, and a potentially viable gauge condition is suggested. The monotonicity properties of M through the examples of closed Bianchi I and IX cosmological spacetimes are also discussed. A potential spectral characterization of these cosmological spacetimes, in terms of the spectrum of the Riemannian Dirac operator and the Sen-Witten and the 3-surface twistor operators, is also indicated.
\end{abstract}

\section{Introduction}

In the present paper we discuss three, apparently independent issues: total masses and mass bounds, the spectral properties of certain differential operators, and gauge conditions on closed spacelike hypersurfaces. (A detailed discussion of these problems will be given in the following three subsections of the introduction, where we also formulate our specific questions.) However, it turned out that these questions are not independent, and our results provide common generalizations of previous particular ones. We review the key techniques and results (which have already appeared in [20]) in sections 2 5, and, in section 3, we generalize our previous result on the zero-mass spacetime configuration: the total mass density is zero if and only if the spacetime is holonomically trivial (and not only locally flat) with toroidal spatial topology. Then, in section 6, we illustrate these in the Bianchi I. and FRW spacetimes, and, in section 17, we summarize the message of this contribution.

Here we use the abstract index formalism, and our sign conventions are those of [14]. In particular, the signature of the spacetime metric is $(+,-,-,-)$, and the curvature tensor is defined by $-R_{b c d}^{a} X^{b} V^{c} W^{d}:=V^{c} \nabla_{c}\left(W^{d} \nabla_{d} X^{a}\right)-W^{c} \nabla_{c}\left(V^{d} \nabla_{d} X^{a}\right)-[V, W]^{c} \nabla_{c} X^{a}$ for 
any vector fields $X^{a}, V^{a}$ and $W^{a}$. Thus, Einstein's equations take the form $G_{a b}:=$ $R_{a b}-\frac{1}{2} R g_{a b}=-\kappa T_{a b}$, where $\kappa:=8 \pi G$ and $G$ is Newton's gravitational constant.

\subsection{Total masses}

It is well known that, as a consequence of the principle of equivalence, there is no well defined notion of gravitational energy-momentum density. On the other hand, for spacetimes which are asymptotically flat at spatial or null infinity, there are well defined notions of total energy-momentum of the localized matter + gravity system. These are the Arnowitt-Deser-Misner (ADM) [1] and the Bondi-Sachs (BS) [3, 17, 5] energy-momenta, respectively. They are given by two-surface integrals on spheres at the spatial and (e.g. future) null infinity, rather than integrals of some local expression on spacelike hypersurfaces extending to the spatial and null infinity, respectively. The corresponding total masses are defined as their Lorentzian length.

However, the form of these total energy-momentum expressions is rather different, and, in fact, completely different formalism and techniques are used to analyze the behaviour of the fields and the gravitation in the two asymptotic zones. Hence it is natural to search for a unified mathematical formulation of the two concepts of total energymomentum.

This unified form can be based on an appropriate superpotential $u(K)_{a b}$, which is a differential 2-form depending on some vector field $K^{a}$, such that in the so-called superpotential equation,

$$
\nabla_{[a} u(K)_{b c]}=J(K)_{a b c}+\frac{1}{2} \kappa K^{f} T_{f e} \frac{1}{3 !} \varepsilon^{e}{ }_{a b c},
$$

the current 3-form $J(K)_{a b c}$ is a homogeneous quadratic expression of the first derivative of the gravitational field variables. Here $\varepsilon_{a b c d}$ is the spacetime volume 4-form, and we used Einstein's equations. Then, for any smooth spacelike hypersurface $\Sigma$ with smooth boundary $\mathcal{S}:=\partial \Sigma$, the 'conserved quantity' generated by the spacetime vector field $K^{a}$ is defined by

$$
\mathrm{Q}[K]:=\frac{2}{\kappa} \oint_{\partial \Sigma} u(K)_{a b}=\int_{\Sigma}\left(\frac{2}{\kappa} J(K)_{a b c}+K^{f} T_{f e} \frac{1}{3 !} \varepsilon^{e}{ }_{a b c}\right) .
$$

(If $\Sigma$ extends to the spatial or future null infinity in an asymptotically flat spacetime, then the integral on its boundary at infinity is defined in a limiting procedure.) Thus the key question is how to choose the generator vector field $K^{a}$ and the superpotential $u(K)_{a b}$ to recover the ADM/BS 4-momenta in the form $\mathrm{Q}[K]$ ?

This question was addressed (among others) by Horowitz and Tod in [9]. They showed that a particularly successful strategy is to use two-component spinors, and to choose the generator vector field to be $K^{a}=\lambda^{A} \bar{\lambda}^{A^{\prime}}$ and the superpotential to be the Nester-Witten 2 -form

$$
u(\lambda)_{a b}:=\frac{\mathrm{i}}{2}\left(\bar{\lambda}_{A^{\prime}} \nabla_{B B^{\prime}} \lambda_{A}-\bar{\lambda}_{B^{\prime}} \nabla_{A A^{\prime}} \lambda_{B}\right),
$$

where the spinor field $\lambda^{A}$ is still not specified. (The original form of the superpotential 2 -form that Witten [22] and Nester [11] used was given in terms of Dirac spinors. The above form in terms of Weyl spinors was introduced by Horowitz and Tod [9].) To recover the ADM/BS energy-momenta the spacelike hypersurface $\Sigma$ should be chosen to 
be asymptotically flat/asymptotically hyperboloidal, and the spinor field $\lambda^{A}$ should tend to its own asymptotic value $\infty_{\infty} \lambda^{A}$ in an appropriate order. In addition, the asymptotic value ${ }_{\infty} \lambda^{A}$ should be constant with respect to the two dimensional Sen type connection (see [18]), or should solve the asymptotic twistor equation of Bramson [4, respectively, on the metric 2-spheres at infinity. The geometric meaning of these boundary conditions is that these spinor fields are the spinor constituents of the asymptotic translations at spatial and null infinity, respectively. The space of solutions of these equations form a two-complex dimensional spin space. If $\left\{\varepsilon_{\underline{A}}^{A}\right\}, \underline{A}=0,1$, is a normalized spin frame in these solution spaces, and $\infty_{\infty} \lambda^{\underline{A}}$ denotes the components of $\infty_{\infty} \lambda^{A}$ in this frame, then the components $\mathrm{P}_{\underline{a}}, \underline{a}=0,1,2,3$, of the total energy-momenta can be recovered as

$$
\mathrm{P}_{\underline{a}} \sigma_{\underline{A}}^{\underline{a}} \underline{A}^{\prime} \infty \lambda^{\underline{A}} \bar{\lambda}^{\lambda^{A}}{ }^{\prime}:=\frac{2}{\kappa} \lim _{r \rightarrow \infty} \oint_{\mathcal{S}_{r}} u(\lambda)_{c d},
$$

where $\sigma_{\underline{A}}^{\underline{a}} \underline{A}^{\prime}$ are the standard $S L(2, \mathbb{C})$ Pauli matrices. (Thus, while Latin indices are abstract tensor or spinor indices, the underlined and (below) the boldface Latin indices are concrete name indices, taking numerical values.)

Let $E_{\underline{a}}^{a}:=\varepsilon_{\underline{A}}^{A} \bar{\varepsilon}_{\underline{A}^{\prime}}^{A^{\prime}} \sigma_{\underline{a}} \underline{A}^{\prime}$, the orthonormal vector basis determined by this spinor dyad. If on the asymptotically flat $\Sigma$ the basis $\left\{E_{a}^{a}\right\}$ is chosen to be adapted to the hypersurface $\Sigma$ (in the sense that $E_{0}^{a}$ is just the future pointing timelike normal of $\Sigma$ ), then the traditional ADM energy $E$ and linear momentum $P_{\mathbf{i}}, \mathbf{i}=1,2,3$, can be recovered as the time and spacelike components, respectively, of $\mathrm{P}_{\underline{a}}$. If, in addition, the spinor field $\lambda^{A}$ is chosen to be normalized at infinity with respect to the timelike normal of $\Sigma$, i.e. $\infty_{\infty} t_{A A^{\prime} \infty} \lambda^{A}{ }_{\infty} \bar{\lambda}^{A^{\prime}}=1$, then the left hand side of (1.4) has the structure $\mathrm{E}+\mathrm{P}_{\mathbf{i}} v^{\mathbf{i}}$, where $v^{\mathbf{i}} v^{\mathbf{j}} \delta_{\mathbf{i j}}=1$. We will need this form of the left hand side of equation (1.4).

Similarly, if on the asymptotically hyperboloidal $\Sigma$ the spin frame $\left\{\varepsilon_{\underline{A}}^{A}\right\}$ in the solution space of the asymptotic twistor equation is chosen such that the vector $E_{0}^{a}=\varepsilon_{\underline{A}}^{A} \bar{\varepsilon}_{A^{\prime}}^{A^{\prime}} \sigma_{0}^{\underline{A}} \underline{A}^{\prime}$ is the BMS time translation of the future null infinity, then the BS energy and linear momentum are just the time and spacelike components, respectively, of $\mathrm{P}_{\underline{a}}$. If, in addition, the spinor field is normalized such that $\sigma_{\underline{A} \underline{A}^{\prime} \infty}^{0} \lambda^{\underline{A}} \bar{\lambda}^{\underline{A}^{\prime}}=1$, then the left hand side of (1.4) has the structure $\mathrm{E}+\mathrm{P}_{\mathrm{i}} v^{\mathbf{i}}$.

Then the positive energy theorems (see [22, 11, 15, 9, 16, 13]) guarantee that the total energy-momenta, both the ADM and the $\mathrm{BS}$, are future pointing and timelike with respect to the natural Lorentzian metric $\eta_{\underline{a} \underline{b}}:=\operatorname{diag}(1,-1,-1,-1)$ coming from the symplectic scalar product of the solution spaces, provided the dominant energy condition is satisfied on the regular, asymptotically flat and asymptotically hyperboloidal $\Sigma$, respectively. Introducing the total mass $\mathrm{m}$ according to $\mathrm{m}^{2}:=\eta_{\underline{a} \underline{b}} \mathrm{P}^{\underline{a}} \mathrm{P} \underline{b}$, the positive energy theorems can be restated as $\mathrm{m} \geq 0$. The rigidity part of these theorems guarantees that if the total mass is zero, $\mathrm{m}=0$, then the domain of dependence of $\Sigma$ is flat.

The mass positivity results motivate the question whether we can find a strictly positive lower bound for the total (ADM and BS) masses, i.e. that $\mathrm{m}^{2} \geq \mathrm{M}^{2}>0$ holds for some M. Another question came from a recent result of Bäckdahl and Valiente-Kroon [2]. They showed by explicit calculation that in vacuum, asymptotically flat spacetimes the ADM mass can be expressed as the $L_{2}$-norm on some spacelike hypersurface of the 3 -surface twistor derivative of an appropriate spinor field: $\mathrm{m}_{A D M}=$ const. $\left\|\mathcal{D}_{(A B} \lambda_{C)}\right\|_{L_{2}}^{2}$. Thus the question is whether we can have a similar expression for $\mathrm{m}_{B S}$, too, and how this result can be generalized for the non-vacuum case.

Since the expected general form of the energy-momentum in General Relativity is a 
two-surface integral, it does not seem to be possible to associate any well defined notion of total energy-momentum, or at least total mass, with closed universes. However, this does not mean a priori that a reasonable and useful notion of total mass cannot be associated with closed universes in some other way. This could perhaps be based on the idea that the total mass should be some positive definite measure of the strength of the gravitational 'field'.

\subsection{Spectral characterization of geometries}

A potentially viable strategy to characterize Riemannian manifolds in an invariant way could be based on the study of the (structure of the) spectrum of elliptic (e.g. Laplace, Dirac, etc.) operators. The key idea is that e.g. the eigenvalues of elliptic operators encode (maybe in some highly non-explicit, but invariant way) certain properties of the geometry, and in the ideal case the whole geometry could be hoped to be characterized completely by the spectrum of a sufficiently large number of elliptic operators. Thus first it should be clarified e.g. how the eigenvalues reflect the properties of the geometry.

The first who obtained such a link between the eigenvalues and certain properties of the geometry was probably Lichnerowicz [10]. He showed that on closed $m$ dimensional spin manifolds $M$ with non-negative scalar curvature $R$ for the 1st eigenvalue of the Dirac operator, $\mathrm{i} \gamma_{e}^{\alpha}{ }_{\beta} D^{e} \Psi^{\beta}=\alpha_{1} \Psi^{\alpha}$, one has $\alpha_{1}^{2} \geq \frac{1}{4} \inf \{R(p) \mid p \in M\}$. This lower bound is, however, not sharp. Lichnerowicz's bound was increased by Friedrich [7] by giving the sharp lower bound: $\alpha_{1}^{2} \geq \frac{m}{4(m-1)} \inf \{R(p) \mid p \in M\}$. This bound is, in fact, saturated by the metric spheres. Later, several other sharp bounds were derived under various geometrical conditions.

From the point of view of General Relativity it would be desirable to extend the above results from Riemannian manifolds to initial data sets. In fact, Hijazi and Zhang [8] derived a sharp lower bound for the 1st eigenvalue of the Sen-Witten operator on closed hypersurfaces in Lorentzian geometries. In terms of the standard notions in GR their bound is

$$
\alpha_{1}^{2} \geq \frac{3}{4} \kappa \inf _{l^{a}} \frac{\int_{\Sigma} t^{a} T_{a b} l^{b} \mathrm{~d} \Sigma}{\int_{\Sigma} t_{c} l^{c} \mathrm{~d} \Sigma},
$$

where the infimum is taken on the set of all the future pointing null vector fields $l^{a}$ on the hypersurface. Thus the bound is the infimum of an average of the total matter energy in $\Sigma$. (The bound of Hijazi and Zhang was rediscovered independently in [19], and the form (1.5) of their bound is taken from [19].)

Nevertheless, the lower bound (1.5) is zero in vacuum, giving no restriction on the eigenvalues. This motivates the question whether we can find an even greater, and hence sharp, lower bound which is not trivial even in vacuum. A more ambitious claim is to derive an expression for the first eigenvalue itself, rather to have only a lower bound for it.

\subsection{Gauge conditions}

In various specific problems of General Relativity (e.g. in the energy positivity proofs, evolution problems, numerical calculations, etc) it is desirable to reduce the huge gauge freedom of the theory. These conditions are used to single out some 'preferred' frame of reference, which are built from a special spinor field (see e.g. [6]). Such conditions 
are, for example, the Witten [22], the Parker [13] and the Nester [12] gauge conditions; and the so-called approximate twistor equation of Bäckdahl and Valiente-Kroon [2] can also be interpreted as a gauge condition. In the present contribution we discuss only the gauge conditions of Witten and of Bäckdahl and Valiente-Kroon.

Witten's gauge condition is simply the differential equation $\mathcal{D}_{A^{\prime}{ }_{A}} \lambda^{A}=0$ for the spinor field on $\Sigma$ which satisfies some boundary condition, where $\mathcal{D}_{a}:=P_{a}^{b} \nabla_{b}$ is the projection to $\Sigma$ of the spacetime Levi-Civita derivative operator $\nabla_{a}$, known as the Sen connection. Similarly, the approximate twistor equation on $\Sigma$ is $\mathcal{T}^{*} \mathcal{T}(\lambda)=0$, which is the composition of the 3-surface twistor operator $\mathcal{T}$ of Tod [21] and its formal adjoint $\mathcal{T}^{*}$. The former is defined to be the totally symmetric part of the derivative, i.e. $\mathcal{T}: \lambda_{A} \mapsto \mathcal{D}_{\left({ }_{A B} \lambda_{C)}\right.}$, where $\mathcal{D}_{A B}$ is the unitary spinor form of $\mathcal{D}_{A A^{\prime}}$ (see e.g. [15]). The formal adjoint $\mathcal{T}^{*}$ is defined with respect to the $L_{2}$ scalar product, and we give its explicit form by (5.1) in section 5. It is known that both the Witten and the approximate twistor equations admit non-trivial solutions on asymptotically flat hypersurfaces. (For the proofs see e.g. [16] and [2], respectively. The existence of solutions of the Witten equation on asymptotically hyperboloidal hypersurfaces was also demonstrated in [16].)

Nevertheless, as far as we know, it has not been clarified whether these gauge conditions admit non-trivial solutions on closed spacelike hypersurfaces. Hence it is natural to ask whether these gauge conditions can be imposed in closed universes, and if not, then how they can be modified to obtain an appropriate one.

\section{The norm identity}

In our investigations the key geometric ingredient is the Reula-Tod (or $S L(2, \mathbb{C})$ spinor) form [16] of the Sen-Witten identity:

$$
\begin{aligned}
& D_{a}\left(t^{A^{\prime} B} \bar{\lambda}^{B^{\prime}} \mathcal{D}_{B B^{\prime}} \lambda^{A}-\bar{\lambda}^{A^{\prime}} t^{A B^{\prime}} \mathcal{D}_{B^{\prime} B} \lambda^{B}\right)+2 t^{A A^{\prime}}\left(\mathcal{D}_{A B^{\prime}} \bar{\lambda}^{B^{\prime}}\right)\left(\mathcal{D}_{A^{\prime} B} \lambda^{B}\right) \\
& \quad=-t_{A A^{\prime}} h^{e f}\left(\mathcal{D}_{e} \lambda^{A}\right)\left(\mathcal{D}_{f} \bar{\lambda}^{A^{\prime}}\right)+\frac{1}{2} \kappa t^{a} T_{a b} \lambda^{B} \bar{\lambda}^{B^{\prime}}
\end{aligned}
$$

where $t^{a}$ is the future pointing unit timelike normal of $\Sigma, P_{b}^{a}=\delta_{b}^{a}-t^{a} t_{b}$ the projection to $\Sigma$, and $h_{a b}:=P_{a}^{c} P_{b}^{d} g_{c d}$ is the induced negative definite metric on $\Sigma$. This equation is just the Hodge dual of the pull back to $\Sigma$ of the superpotential equation (1.1), in which the superpotential is the Nester-Witten 2-form, and Einstein's equation is used. The total divergence on the left is just the Hodge dual of the pull back to $\Sigma$ of the exterior derivative of $u(\lambda)_{a b}$.

The key observation is that the (algebraically) irreducible decomposition of the unitary spinor form [15] of the $\mathcal{D}_{e}$-derivative of the spinor field into its totally symmetric part and the traces,

$$
\mathcal{D}_{E F} \lambda_{A}=\mathcal{D}_{(E F} \lambda_{A)}+\frac{2 \sqrt{2}}{3} t_{F}^{E^{\prime}} P_{E E^{\prime}}^{C C^{\prime}} \varepsilon_{C A} \mathcal{D}_{C^{\prime} D} \lambda^{D},
$$

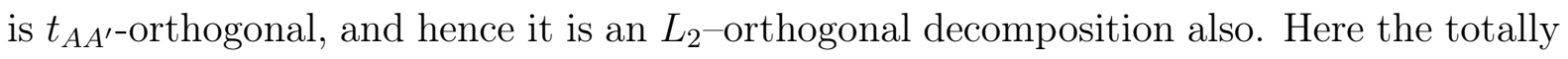
symmetric part of the derivative defines the 3-surface twistor operator, while the second term is proportional to the action of the Sen-Witten operator (i.e. the Dirac operator built from the Sen connection on $\Sigma$ ) on the spinor field. 
Substituting this decomposition into the Sen-Witten identity and taking its integral on $\Sigma$ we obtain

$$
\mathrm{Q}[\lambda \bar{\lambda}]+\frac{4 \sqrt{2}}{3 \kappa}\left\|\mathcal{D}_{A^{\prime} A} \lambda^{A}\right\|_{L_{2}}^{2}=\frac{\sqrt{2}}{\kappa}\left\|\mathcal{D}_{(A B} \lambda_{C)}\right\|_{L_{2}}^{2}+\int_{\Sigma} t^{a} T_{a b} \lambda^{B} \bar{\lambda}^{B^{\prime}} \mathrm{d} \Sigma .
$$

Here $\mathrm{Q}[\lambda \bar{\lambda}]$ is the integral of $\frac{2}{\kappa} u(\lambda)_{a b}$ on the boundary $\partial \Sigma$, and the $L_{2}-$ norm of the spinor field is defined to be the integral on $\Sigma$ of the pointwise Hermitian scalar product $\sqrt{2} t_{A A^{\prime}} \lambda^{A} \bar{\lambda}^{A^{\prime}}$. This equation will play a key role in what follows and we call it the basic norm identity. Clearly, if $\Sigma$ is asymptotically flat or asymptotically hyperboloidal, then the existence of the $L_{2}$ norms and the integral of the energy-momentum tensor require appropriate fall-off properties both for the geometry and the energy-momentum tensor, and also for the spinor fields.

\section{Total masses and mass bounds}

\subsection{The asymptotically flat/asymptotically hyperboloidal cases}

By the expression (1.4) of the ADM/BS energy-momenta, the basic norm identity (2.3) and Witten's gauge condition we obtain the following simple expression for the total energy-momenta:

$$
\mathrm{P}_{\underline{a}} \sigma_{\underline{A}}^{\underline{a}} \underline{A}^{\prime} \infty \lambda^{\underline{A}} \bar{\lambda}^{\underline{A}^{\prime}}=\frac{\sqrt{2}}{\kappa}\left\|\mathcal{D}_{(A B} \lambda_{C)}\right\|_{L_{2}}^{2}+\int_{\Sigma} t^{a} T_{a b} \lambda^{B} \bar{\lambda}^{B^{\prime}} \mathrm{d} \Sigma .
$$

This expression is an extension of the result of Bäckdahl and Valiente-Kroon for the $\mathrm{ADM}$ energy in vacuum, mentioned in subsection 1.1; It gives both the ADM and BS total energy-momenta, even in the non-vacuum case, in terms of the $L_{2}$-norm of the 3 -surface twistor derivative of an appropriate spinor field and the integral of the energymomentum tensor of the matter fields.

The right hand side of (3.1) motivates the introduction of the following quantity:

$$
\mathrm{M}:=\inf \left\{\frac{\sqrt{2}}{\kappa}\left\|\mathcal{D}_{(A B} \lambda_{C)}\right\|_{L_{2}}^{2}+\int_{\Sigma} t^{a} T_{a b} \lambda^{B} \bar{\lambda}^{B^{\prime}} \mathrm{d} \Sigma\right\}
$$

Here the infimum is taken on the set of the spinor fields satisfying the boundary and normalization conditions that we had in the asymptotically flat/asymptotically hyperboloidal cases, respectively.

Let us write the components of the energy-momentum as $\mathrm{P}^{\underline{a}}=\left(\mathrm{E}, \mathrm{P}^{\mathrm{i}}\right)$. Then, by the positive energy theorem $\mathrm{E} \geq\left|\mathrm{P}^{\mathrm{i}}\right|$, for the total mass we obtain that $\mathrm{m}^{2}:=\mathrm{P}^{\underline{a}} \mathrm{P}^{\underline{b}} \eta_{\underline{a}} \underline{b}=$ $\mathrm{E}^{2}-\left|\mathrm{P}^{\mathrm{i}}\right|^{2}=\left(\mathrm{E}-\left|\mathrm{P}^{\mathbf{i}}\right|\right)\left(\mathrm{E}+\left|\mathrm{P}^{\mathbf{i}}\right|\right) \geq\left(\mathrm{E}-\left|\mathrm{P}^{\mathbf{i}}\right|\right)^{2}$, where $\left|\mathrm{P}^{\mathbf{i}}\right|$ denotes the magnitude of the linear momentum $\mathrm{P}^{\mathbf{i}}$. However, since $\mathrm{E}-\left|\mathrm{P}^{\mathbf{i}}\right|=\inf \left\{\mathrm{E}+\mathrm{P}_{\mathbf{i}} v^{\mathbf{i}} \mid v^{\mathbf{i}} v^{\mathbf{j}} \delta_{\mathbf{i j}}=1\right\}=\mathrm{M}$, the expression (3.2) provides a lower bound for $\mathrm{m}$. (Here the infimum is taken on the set of the unit vectors $v^{\mathbf{i}}$.) By the rigidity part of the positive energy theorems (i.e. if $\mathrm{E}=\left|\mathrm{P}^{\mathbf{i}}\right|$ then the spacetime is flat), the lower bound M for the ADM and BS masses is strictly positive, unless the spacetime is flat.

\subsection{The closed case}

If $\Sigma$ is closed, i.e. compact with no boundary, then no total energy-momentum (and hence mass) can be introduced in the form of a two-surface integral. However, the quantity $M$ 
can still be introduced by (3.2) with an appropriately chosen set of spinor fields on which the infimum is taken. Clearly, since $\Sigma$ is closed, we do not have any boundary condition for the spinor fields, but we should impose a normalization condition. The most natural such condition seems to be $\left\|\lambda^{A}\right\|_{L_{2}}^{2}=1$, which was used in [20]. However, the condition $1=\int_{\Sigma} t_{A A^{\prime}} \lambda^{A} \bar{\lambda}^{A^{\prime}} \mathrm{d} \Sigma=\frac{1}{\sqrt{2}}\left\|\lambda^{A}\right\|_{L_{2}}^{2}$ is even more natural, since it is just the integral of the pointwise norm $t_{A A^{\prime}} \lambda^{A} \bar{\lambda}^{A^{\prime}}$ whose asymptotic form, $\infty_{\infty} t_{A A^{\prime} \infty} \lambda^{A}{ }_{\infty} \bar{\lambda}^{A^{\prime}}=1$, was used to normalize the spinor field e.g. at spatial infinity in the asymptotically flat case. Another normalization condition could be $\left\|\lambda^{A}\right\|_{L_{2}}^{2}=\sqrt{2} \operatorname{vol}(\Sigma)$, i.e. the 'average' of the pointwise norm $t_{A A^{\prime}} \lambda^{A} \bar{\lambda}^{A^{\prime}}$ on $\Sigma$ would be required to be 1 . The physical dimension of $\mathrm{M}$ in the two cases is mass-density and mass, respectively. However, although the second choice appears to yield a dimensionally correct mass expression, for later convenience we choose the first normalization condition. Note that with this normalization $\mathrm{M}$ is $\sqrt{2}$-times of the M introduced in [20].

Since $\Sigma$ does not have a boundary, the basic norm identity (2.3) for any spinor field and the definition of $\mathrm{M}$ yield that

$$
\frac{4 \sqrt{2}}{3 \kappa}\left\|\mathcal{D}_{A^{\prime} A} \lambda^{A}\right\|_{L_{2}}^{2}=\left\{\frac{\sqrt{2}}{\kappa}\left\|\mathcal{D}_{(A B} \lambda_{C)}\right\|_{L_{2}}^{2}+\int_{\Sigma} t^{a} T_{a b} \lambda^{B} \bar{\lambda}^{B^{\prime}} \mathrm{d} \Sigma\right\} \geq \frac{1}{\sqrt{2}} \mathrm{M}\left\|\lambda^{A}\right\|_{L_{2}}^{2} .
$$

Since $\mathrm{M}$ was defined as the infimum of an expression on a set of certain smooth spinor fields, it is not a priori obvious that there is a smooth spinor field which saturates the inequality on the right. Nevertheless, one can in fact show that such a spinor field does exist [20]. We will call such a spinor field a minimizer spinor field. Thus, if $\lambda^{A}$ is such a minimizer spinor field and $\langle\cdot, \cdot\rangle$ denotes the $L_{2}$ scalar product, then, for this spinor field, by (3.3) we have that

$$
\left\langle 2 \mathcal{D}^{A A^{\prime}} \mathcal{D}_{A^{\prime} B} \lambda^{B}-\frac{3}{4} \kappa \mathrm{M} \lambda^{A}, \lambda^{A}\right\rangle=2\left\|\mathcal{D}_{A^{\prime} A} \lambda^{A}\right\|_{L_{2}}^{2}-\frac{3}{4} \kappa \mathrm{M}\left\|\lambda^{A}\right\|_{L_{2}}^{2}=0 .
$$

This implies that either the minimizer spinor field is necessarily $L_{2}$-orthogonal to the spinor field $2 \mathcal{D}^{A A^{\prime}} \mathcal{D}_{A^{\prime} B} \lambda^{B}-\frac{3}{4} \kappa \mathrm{M} \lambda^{A}$, or that $\frac{3}{4} \kappa \mathrm{M}$ is an eigenvalue and the minimizer spinor field is a corresponding eigenspinor of the operator $2 \mathcal{D}^{A A^{\prime}} \mathcal{D}_{A^{\prime} B}$. We will see in the next section that this is, indeed, the case, and $\frac{3}{4} \kappa \mathrm{M}$ is its smallest eigenvalue.

The (geometrical and physical) significance of $M$ is shown by the result [20] that the vanishing of $\mathrm{M}$ is equivalent to the flatness of the spacetime with toroidal spatial topology. However, if we allow to have locally flat, but holonomically non-trivial spacetime configurations, then in such domains the constant spinor fields are not necessarily continuous everywhere, and hence our previous theorem should be modified. This possibility motivated the following generalization of our previous result:

Theorem 3.1. Let the matter fields satisfy the dominant energy condition. Then $\mathrm{M}=0$ for some (and hence for any) $\Sigma$ if and only if the spacetime is holonomically trivial and the topology of $\Sigma$ is torus: $\Sigma \approx S^{1} \times S^{1} \times S^{1}$.

Proof. Since the detailed proof (with a different line of argument) of the original statement is given in [20], here we only summarize its key points and concentrate on the difference between the present and the original statements. Clearly, in holonomically trivial spacetime with $\mathbb{R} \times S^{1} \times S^{1} \times S^{1}$ global topology there are globally defined constant spinor fields, which satisfy the 3 -surface twistor equation. Therefore, $\mathrm{M}=0$. 
Conversely, suppose that $M=0$. Then the minimizer spinor field satisfies $\mathcal{D}_{(A B} \lambda_{C)}=0$, and hence by the basic norm identity $\mathcal{D}_{e} \lambda^{A}=0$ follows. This implies that $Z_{a}:=P_{a}^{b} \lambda_{B} \bar{\lambda}_{B^{\prime}}$ is surface-orthogonal, i.e. $Z_{a}=D_{a} u$ for some (locally defined) function $u$ on $\Sigma$. Using the Gauss equation for hypersurfaces one can show that the level sets $\mathcal{S}_{u}:=\{u=$ const $\}$ are locally flat 2 -surfaces in $\Sigma$.

Next, one can show that the level sets $\mathcal{S}_{u}$ are closed surfaces, which by the GaussBonnet theorem are necessarily two-tori. $\Sigma$ is globally foliated by these tori, and hence it is a 3-torus.

Finally, we use the field equations. In an appropriate spacetime coordinate system adapted to the geometry they reduce to a single Poisson equation with a source term on the level sets $\mathcal{S}_{u}$. This source is, however, non-negative by the dominant energy condition. To have a non-trivial solution to this equation both the source term and the not a priori zero components of the curvature have to be zero, i.e. the spacetime is flat. Let $\gamma$ be any smooth homotopically non-trivial closed curve. Then the constant spinor field $\lambda^{A}$ is clearly parallelly propagated along $\gamma$. However, if the holonomy $H_{\gamma}$ were not trivial, then $\lambda^{A}$ could not be continuous everywhere along $\gamma$. Thus the whole holonomy group must be trivial.

By definition, $M$ is non-negative, and hence the content of this theorem is analogous to the rigidity part of the positive energy theorems for the ADM/BS masses. Thus $\mathrm{M}$ is a positive definite measure of the strength of the gravitational field. Since the physical dimension of $\operatorname{Mvol}(\Sigma)$ is mass, moreover it is given by precisely the formula that we had in the previous subsection for the positive lower bound for the ADM/BS masses, it seems plausible to interpret $\mathrm{M}$ as the total mass density of closed universes at the instant represented by the hypersurface $\Sigma$.

\section{The eigenvalue problem}

Since in four dimensions the spinors are the four-component Dirac spinors (see e.g. the appendix of [14]), and the Sen connection from which the Sen-Witten operator is constructed is defined on a vector bundle over $\Sigma$ whose fibers are four dimensional Lorentzian vector spaces, the eigenvalue problem should be formulated in terms of Dirac spinors. (For the discussion of the difficulties with other approaches, see [20].) Thus, the eigenvalue problem for the Sen-Witten operator is defined by $\mathrm{i} \gamma_{e \beta}^{\alpha} \mathcal{D}^{e} \Psi^{\beta}=\alpha \Psi^{\alpha}$, where the Greek indices are abstract indices referring to the space of the Dirac spinors, and $\gamma_{e \beta}^{\alpha}$ are Dirac's ' $\gamma$-matrices'. Recalling that a Dirac spinor $\Psi^{\alpha}$ is a pair $\left(\lambda^{A}, \bar{\mu}^{A^{\prime}}\right)$ of Weyl spinors, with the explicit form of $\gamma_{e \beta}^{\alpha}$ given in [14] the eigenvalue problem is equivalent to the pair

$$
\mathrm{i} \mathcal{D}_{A^{\prime} A} \lambda^{A}=\frac{\alpha}{\sqrt{2}} \bar{\mu}_{A^{\prime}}, \quad \mathrm{i} \mathcal{D}_{A A^{\prime}} \bar{\mu}^{A^{\prime}}=\frac{\alpha}{\sqrt{2}} \lambda_{A}
$$

of equations. Taking the action of $\mathcal{D}^{B A^{\prime}}$ on the first of these equations and eliminating $\bar{\mu}_{A^{\prime}}$ by the second, we obtain that the eigenvalue problem is equivalent to $2 \mathcal{D}^{A A^{\prime}} \mathcal{D}_{A^{\prime} B} \lambda^{B}=$ $\alpha^{2} \lambda^{A}$. Moreover, (4.1) implies that the eigenvalue $\alpha$ is real: $0 \leq 2\left\|\mathcal{D}_{A^{\prime} A} \lambda^{A}\right\|_{L_{2}}^{2}=$ $2\left\langle\mathcal{D}^{A A^{\prime}} \mathcal{D}_{A^{\prime} B} \lambda^{B}, \lambda^{A}\right\rangle=\alpha^{2}\left\|\lambda^{A}\right\|_{L_{2}}^{2}$. Now we show that the multiplicity of every nonzero eigenvalue $\alpha^{2}$ is even. In fact, if $\lambda^{A}$ is an eigenspinor, then $\mu^{A}=-\mathrm{i} \frac{\sqrt{2}}{\alpha} \mathcal{D}^{A}{ }_{A^{\prime}} \bar{\lambda}^{A^{\prime}}$ is also an eigenspinor. But if $\mu^{A}$ were not independent of $\lambda^{A}$, then $\mu^{A}=c \lambda^{A^{\alpha}}$ would hold for some non-zero complex constant $c$. Substituting this back into (4.1) we obtain that 
$\alpha\left(1+|c|^{2}\right) \lambda^{A}=0$, which would contradict $\alpha \neq 0$. Thus, for each Dirac eigenspinor $\Psi^{\alpha}$, we have a pair of independent eigenspinors of $2 \mathcal{D}^{A A^{\prime}} \mathcal{D}_{A^{\prime} B}$.

Applying the basic norm identity to the eigenspinor $\lambda^{A}$ we obtain that $\alpha^{2} \geq \frac{3}{4} \kappa \mathrm{M}$, i.e. $\frac{3}{4} \kappa \mathrm{M}$ is a lower bound for all the eigenvalues of $2 \mathcal{D}^{A A^{\prime}} \mathcal{D}_{A^{\prime} B}$. We will see that this is precisely the smallest eigenvalue $\alpha_{1}^{2}$, i.e. a sharp lower bound. The proof of this statement is based on the following functional analytic properties of the operator:

Theorem 4.1. There is a dense subspace $\operatorname{Dom}\left(\mathcal{D}^{*} \mathcal{D}\right) \subset H_{1}\left(\Sigma, \mathbb{S}^{A}\right)$ of the first Sobolev space of the unprimed spinor fields on $\Sigma$ such that it contains the space $C^{\infty}\left(\Sigma, \mathbb{S}^{A}\right)$ of the smooth spinor fields, and there is an extension of the operator $\mathcal{D}^{A A^{\prime}} \mathcal{D}_{A^{\prime} B}$ from $C^{\infty}\left(\Sigma, \mathbb{S}^{A}\right)$ to $\operatorname{Dom}\left(\mathcal{D}^{*} \mathcal{D}\right)$ such that $\mathcal{D}^{A A^{\prime}} \mathcal{D}_{A^{\prime} B}: \operatorname{Dom}\left(\mathcal{D}^{*} \mathcal{D}\right) \rightarrow L_{2}\left(\Sigma, \mathbb{S}^{A}\right)$ is a positive self-adjoint Fredholm operator with compact resolvent.

There is a similar result for the composition $\mathcal{T}^{*} \mathcal{T}$ of the 3 -surface twistor operator and its adjoint, too. The detailed proof of these statements is given in the Appendix of [20].

The significance of this theorem is that, via standard theorems of functional analysis, it guarantees that (1) the spectrum of $2 \mathcal{D}^{A A^{\prime}} \mathcal{D}_{A^{\prime} B}$ (and of $2 \mathcal{T}^{*} \mathcal{T}$, too) is purely discrete, and that (2) the corresponding eigenspinors span the whole space $L_{2}\left(\Sigma, \mathbb{S}^{A}\right)$. Thus we can order the eigenvalues into the increasing sequence $\alpha_{1}^{2} \leq \alpha_{2}^{2} \leq \cdots \leq \alpha_{i}^{2} \leq \cdots$, and the sequence of the corresponding independent eigenspinors are denoted by $\left\{\lambda_{i}^{A}\right\}$. (Because of the multiplicity of the eigenvalues we should allow equality in the sequence of the eigenvalues.) Clearly, the eigenspinors $\left\{\lambda_{i}^{A}\right\}$ can be chosen to form an $L_{2}$-orthogonal system.

Then let us expand the minimizer spinor field as $\lambda^{A}=\sum_{i} c_{i} \lambda_{i}^{A}$, where $c_{i} \in \mathbb{C}$. Substituting this form of $\lambda^{A}$ into (3.4) we find

$$
0=\left\langle 2 \mathcal{D}^{A A^{\prime}} \mathcal{D}_{A^{\prime} B} \lambda^{B}-\frac{3}{4} \kappa \mathrm{M} \lambda^{A}, \lambda^{A}\right\rangle=\sum_{i}\left|c_{i}\right|^{2}\left(\alpha_{i}^{2}-\frac{3}{4} \kappa \mathrm{M}\right)\left\|\lambda_{i}^{A}\right\|_{L_{2}}
$$

Taking into account that $\frac{3}{4} \kappa \mathrm{M}$ is a lower bound for all the eigenvalues $\alpha^{2}$, we conclude that

$$
\alpha_{1}^{2}=\frac{3}{4} \kappa \mathrm{M}
$$

otherwise $\lambda^{A}$ would have to be zero. Therefore, the total mass (density) of closed universes can be recovered as the first eigenvalue of the Sen-Witten operator, which result makes $M$ a well computable quantity. Looking at (4.3) from the point of view of the spectral characterization of geometries, it is an explicit formula for the first eigenvalue of the SenWitten operator rather than only a lower bound for it. Hence (4.3) is a generalization of the result [8] of Hijazi and Zhang.

\section{On the gauge conditions}

An immediate consequence of the basic norm identity and Theorem 3.1 is that Witten's gauge condition admits non-trivial solution if and only if $\mathrm{M}=0$, i.e. precisely when the spacetime is holonomically trivial with toroidal $\Sigma$.

To discuss the existence of solutions to the approximate twistor equation we need the explicit form of $\mathcal{T}^{*} \mathcal{T}$. First, the formal adjoint of the 3-surface twistor operator, $\mu_{A B C} \mapsto \mathcal{T}^{*}(\mu)_{A}, \mu_{A B C}=\mu_{(A B C)}$, is 


$$
\mathcal{T}^{*}(\mu)_{A}={ }^{+} \mathcal{D}^{B C} \mu_{A B C},
$$

where ${ }^{+} \mathcal{D}_{A B} \lambda_{C}:=D_{A B} \lambda_{C}-\frac{1}{\sqrt{2}} \chi_{A B C}{ }^{D} \lambda_{D}$ and $\chi_{A B C D}$ is the unitary spinor form of the extrinsic curvature. Then, comparing the explicit form of $\mathcal{T}^{*} \mathcal{T}$ with that of $\mathcal{D}^{A A^{\prime}} \mathcal{D}_{A^{\prime} B}$ (given e.g. in terms of the Levi-Civita derivative operator $D_{a}$ and the extrinsic curvature $\left.\chi_{a b}\right)$, we find that

$$
\mathcal{T}^{*} \mathcal{T}(\lambda)_{A}=\frac{4}{3} \mathcal{D}_{A A^{\prime}} \mathcal{D}^{A^{\prime} B} \lambda_{B}-\kappa t_{e} T^{e A^{\prime} B} t_{A^{\prime} A} \lambda_{B}
$$

Thus, apart from a zeroth order operator, the approximate twistor operator is essentially the square of the Sen-Witten operator. Since the kernel of the Sen-Witten and the square of the Sen-Witten operators coincide, this implies that in vacuum it does not admit any solution.

On the other hand, the results of section 4 suggest a potentially viable alternative gauge condition. Namely, let us choose the eigenspinors of the Sen-Witten operator corresponding to the first eigenvalue. We showed in section 4 that there exist at least two such linearly independent spinor fields. Our conjecture is that the eigenspinors with the first eigenvalue can have no zeros, and hence, in particular, the number of these eigenspinors is precisely two. (In fact, if there were three such linearly independent eigenspinors, say $\lambda_{1}^{A}, \lambda_{2}^{A}$ and $\lambda_{3}^{A}$, then, since the spin space is two-complex dimensional, for any point $p \in \Sigma$ there would be non-zero complex constants $c_{1}, c_{2}$ and $c_{3}$ such that $c_{1} \lambda_{1}^{A}(p)+c_{2} \lambda_{2}^{A}(p)+c_{3} \lambda_{3}^{A}(p)=0$ would hold, i.e. the eigenspinor $\lambda^{A}:=c_{1} \lambda_{1}^{A}+c_{2} \lambda_{2}^{A}+c_{3} \lambda_{3}^{A}$ would have a zero at $p$.) Therefore, this gauge condition would yield a geometrically distinguished 3-parameter family of globally defined orthonormal vector bases and lapse functions on $\Sigma$.

\section{$6 \quad$ Examples}

\subsection{Bianchi I. spacetimes}

Let $\Sigma$ be a $t=$ const hypersurface in the Bianchi I. cosmological model with toroidal spatial topology. The induced intrinsic metric on $\Sigma$ is flat, and let us write the corresponding line element as $d h^{2}=-\left(a^{2} d \psi^{2}+b^{2} d \theta^{2}+c^{2} d \phi^{2}\right)$, where $a, b, c \in(0, \infty)$ and the coordinates are $\psi, \theta, \phi \in[0,2 \pi)$. In the global orthonormal basis adapted to the spatial symmetries the extrinsic curvature of $\Sigma$ can be written as $\chi_{a b}=\operatorname{diag}\left(\chi_{1}, \chi_{2}, \chi_{3}\right)$, where the diagonal elements are constant on $\Sigma$.

First, we calculate the spectrum of three differential operators. The simplest one is the square of the Riemannian Dirac operator, built from the intrinsic (flat) Levi-Civita connection $D_{e}$. Since the Sen-Witten operator reduces to the Riemannian Dirac operator when the extrinsic curvature is vanishing, it seems natural to define its eigenvalue problem by $2 D^{A A^{\prime}} D_{A^{\prime} B} \lambda^{B}=\beta^{2} \lambda^{A}$. Then elementary calculations yield its spectrum:

$$
\beta= \pm \sqrt{\left(\frac{n_{1}}{a}\right)^{2}+\left(\frac{n_{2}}{b}\right)^{2}+\left(\frac{n_{3}}{c}\right)^{2}}, \quad n_{1}, n_{2}, n_{3} \in \mathbb{Z},
$$

Thus, from the first few eigenvalues of the Riemannian Dirac operator we can recover the constants $a, b$ and $c$, i.e. the spatial geometry $\left(\Sigma, h_{a b}\right)$ can be characterized completely by the spectrum of the Riemannian Dirac operator. 
Since $\chi_{a b}$ is constant on $\Sigma$, it is easy to see that $2 \mathcal{D}^{A A^{\prime}} \mathcal{D}_{A^{\prime} B} \lambda^{B}=2 D^{A A^{\prime}} D_{A^{\prime} B} \lambda^{B}+$ $\frac{1}{4} \chi^{2} \lambda^{A}$. Therefore, if the eigenvalue problem for the Sen-Witten operator is defined by $2 \mathcal{D}^{A A^{\prime}} \mathcal{D}_{A^{\prime} B} \lambda^{B}=\alpha^{2} \lambda^{A}$ (as in section 4), then

$$
\alpha^{2}=\beta^{2}+\frac{1}{4} \chi^{2}
$$

Thus the spatial geometry and the mean extrinsic curvature can be characterized completely by the spectrum of the Sen-Witten operator.

Finally, we define the eigenvalues of the 3 -surface twistor operator by $2 \mathcal{T}^{*} \mathcal{T}(\lambda)_{A}=$ $\tau^{2} \lambda_{A}$, where the adjoint $\mathcal{T}^{*}$ of the 3 -surface twistor operator has been given explicitly by (5.1). For its eigenvalues we obtain that

$$
\tau^{2}=\frac{4}{3} \beta^{2}+\frac{1}{2}\left(\chi_{a b}-\frac{1}{3} \chi h_{a b}\right)\left(\chi^{a b}-\frac{1}{3} \chi h^{a b}\right) .
$$

Hence the spatial geometry and the magnitude of the trace free part of $\chi_{a b}$ can be characterized completely by the spectrum of the 3-surface twistor operator. It could be interesting to see whether, in addition to the trace of $\chi_{a b}$ and of $\chi_{a c} \chi_{b}^{c}$, the trace of the cube of the extrinsic curvature also can be recovered from the spectrum of some additional (probably higher order) elliptic operator. In this case we would have a complete characterization of the initial data sets for the geometry of the closed Bianchi I. cosmological spacetimes.

Next, let us calculate the total mass density in the closed Bianchi I. cosmological model. Since by the Hamiltonian constraint $\chi^{2}=2 \kappa \mu+\chi_{a b} \chi^{a b}$, by (4.3) and (6.2) it is

$$
\mathrm{M}=\frac{1}{3 \kappa} \chi^{2}=\mu+\frac{1}{2 \kappa}\left(\chi_{a b}-\frac{1}{3} \chi h_{a b}\right)\left(\chi^{a b}-\frac{1}{3} \chi h^{a b}\right) .
$$

Thus the anisotropy of the extrinsic curvature, which is essentially the first eigenvalue of the 3-surface twistor operator, contributes to M. Equation (6.4) illustrates how Theorem 3.1 works: $\mathrm{M}=0$, together with the Hamiltonian constraint, really imply flatness.

We calculate the time derivative of $M$ with respect to an evolution vector field compatible with the spacetime symmetries, $K^{a}=N t^{a}$, where $N$ is constant on $\Sigma$. It is

$$
\dot{\mathrm{M}}=N \chi\left(\frac{1}{3} h^{a b} \sigma_{a b}-\mu-\frac{1}{\kappa}\left(\chi_{a b}-\frac{1}{3} \chi h_{a b}\right)\left(\chi^{a b}-\frac{1}{3} \chi h^{a b}\right)\right),
$$

where $\sigma_{a b}:=P_{a}^{c} P_{b}^{d} T_{c d}$, the spatial stress of the matter fields, and whose trace gives the average (isotropic) pressure: $p=-\frac{1}{3} h^{a b} \sigma_{a b}$. In the 'mean expanding phase' (i.e. when $\chi>0$ ) with 'normal' matter (i.e. $\mu, p \geq 0$ ) the total mass density $\mathrm{M}$ is decreasing. Similarly, we can compute the time derivative of the total mass $\operatorname{Mvol}(\Sigma)$, too. In the 'mean expanding phase' it is also negative. This behaviour is compatible with the interpretation that $\mathrm{M}$ is a positive definite measure of the strength of the gravitational field.

\subsection{FRW spacetimes}

In the initial data set for a closed Friedman-Robertson-Walker spacetime there are only two independent geometrical quantities, the spatial scalar curvature $\mathcal{R}$ and the trace $\chi$ of the extrinsic curvature. These can be recovered from the first eigenvalue of the Riemannian Dirac and of the Sen-Witten operator, respectively: $\beta_{1}^{2}=\frac{3}{8} \mathcal{R}, \alpha_{1}^{2}=\beta_{1}^{2}+\frac{1}{4} \chi^{2}$.

To calculate the total mass density we need the Hamiltonian constraint. It is $\frac{1}{2} \mathcal{R}+$ $\frac{1}{3} \chi^{2}=\kappa \mu$. Thus 


$$
\mathrm{M}=\mu, \quad \dot{\mathrm{M}}=\frac{1}{3} N \chi\left(h^{a b} \sigma_{a b}-3 \mu\right) .
$$

In the expanding phase (i.e. when $\chi>0$ ) with 'normal' matter both $\mathrm{M}$ and $\operatorname{Mvol}(\Sigma)$ are decreasing.

\section{Summary}

The quantity M, defined by equation (3.2) with the set of smooth spinor fields satisfying appropriate boundary conditions at infinity on asymptotically flat or asymptotically hyperboloidal hypersurfaces, provides a positive lower bound for the ADM and Bondi-Sachs masses, respectively.

On closed hypersurfaces for the same M (defined with a different set of the spinor fields) the following statements have been proven:

- $\mathrm{M}=0$ iff the spacetime is holonomically trivial with toroidal Cauchy hypersurface,

- M gives the first eigenvalue $\alpha_{1}^{2}$ of the square $2 \mathcal{D}^{A A^{\prime}} \mathcal{D}_{A^{\prime} B}$ of the Sen-Witten operator:

$$
\alpha_{1}^{2}=\frac{3}{4} \kappa \mathrm{M}
$$

- Witten's gauge condition, $\mathcal{D}_{A^{\prime} A} \lambda^{A}=0$, admits a non-trivial solution iff $\mathrm{M}=0$.

Here we showed that in general the so-called approximate twistor operator cannot be used to determine a gauge condition in closed universes. Nevertheless, we suggested an alternative gauge condition, viz. the use of the eigenspinors of $2 \mathcal{D}^{A A^{\prime}} \mathcal{D}_{A^{\prime} B}$ corresponding to the first eigenvalue above.

Through simple examples we illustrated how the geometry of the data sets for closed universes could be characterized by the spectrum of the Sen-Witten and the 3-surface twistor operators. In these examples we also calculated the quantity $\mathrm{M}$ and its time derivative. The results support the interpretation of $\mathrm{M}$, suggested the general properties listed above: It, as a positive definite measure of the strength of the gravitational 'field', can be interpreted as the total mass density of closed universes at the instant represented by the closed hypersurface $\Sigma$. Nevertheless, the ultimate answer to the question whether this is a reasonable and useful notion will be given by the future applications.

I would like to thank the organizers for the invitation to the Spanish Relativity Meeting in Portugal at Guimarães, 2012 September, where these results could be presented.

\section{References}

[1] R. Arnowitt, S. Deser, C. W. Misner, The dynamics of general relativity, In: Witten, L. (ed) Gravitation: An Introduction to Current Research, pp. 227-265. Wiley, New York, London (1962), online version: gr-qc/0405109

[2] T. Bäckdahl, J. A. Valiente-Kroon, Approximate twistors and positive mass, Class. Quantum Grav. 28075010 (2011), online version: arXiv:1011.3712 [gr-qc] 
[3] H. Bondi, M. G. J. van der Burg, A. W. K. Metzner, Gravitational waves in general relativity. VII. Waves from axi-symmetric isolated systems, Proc. R. Soc. London, Ser. A 269 21-52 (1962)

[4] B. D. Bramson, The alignment of frames of reference at null infinity for asymptotically flat Einstein-Maxwell manifolds, Proc. R. Soc. London, Ser. A 341 451-461 (1975)

[5] A. R. Exton, E. T. Newman, R. Penrose, Conserved quantities in the EinsteinMaxwell theory, J. Math. Phys. 10 1566-1570 (1969)

[6] J. Frauendiener, Triads and the Witten equation, Class. Quantum Grav. 8 1881-7 (1991)

[7] T. Friedrich, Der erste Eigenwert des Dirac-operators einer kompakten Riemannschen Mannigfaltigkeit nichtnegativer Skalarkrümmung, Math. Nachr. 97 117$146(1980)$

[8] O. Hijazi, X. Zhang, The Dirac-Witten operators on spacelike hypersurfaces, Anal. Geom. 11 737-750 (2003)

[9] G. Horowitz, K. P. Tod, A relation between local and total energy in general relativity, Commun. Math. Phys. 85 429-447 (1982)

[10] A. Lichnerowicz, Spineurs harmoniques, C. R. Acad. Sci. Paris A-B 257 7-9 (1963)

[11] J. M. Nester, A new gravitational energy expression with a simple positivity proof, Phys. Lett. A 83 241-42 (1981)

[12] J. M. Nester, A gauge condition for orthonormal three-frames, J. Math. Phys. 30 624-626 (1989)

[13] T. H. Parker, Gauge choice in Witten's energy expression, Commun. Math. Phys. $100471-480(1985)$

[14] R. Penrose, W. Rindler, Spinors and Spacetime, vol 2, Cambridge University Press, Cambridge (1986)

[15] O. Reula, Existence theorem for solutions of Witten's equation and nonnegativity of total mass, J. Math. Phys. 23 810-4 (1982)

[16] O. Reula, K. P. Tod, Positivity of the Bondi energy, J. Math. Phys. 25 1004-8 (1984)

[17] R. K. Sachs, Asymptotic symmetries in gravitational theory, Phys. Rev. 128 2851$2864(1962)$

[18] L. B. Szabados, Two dimensional Sen connections in general relativity, Class. Quantum Grav. 11 1833-1846 (1994), online version: gr-qc/9402005

[19] L. B. Szabados, A lower bound for the eigenvalue of the Sen-Witten operator on closed spacelike hypersurfaces, online version: arXiv:0712.2785 [gr-qc] 
[20] L. B. Szabados, Mass, gauge conditions and spectral properties of the Sen-Witten and 3-surface twistor operators in closed universes, Class. Quantum Grav. 29095001 (30pp) (2012), online version: arXiv:1112.2966 [gr-qc]

[21] K. P. Tod, Three-surface twistors and conformal embedding, Gen. Rel. Grav. 16 435-443 (1984)

[22] E. Witten, A new proof of the positive energy theorem, Commun. Math. Phys. 30 381-402 (1981) 